\title{
Spontaneous rupture of extensor pollicis longus tendon in a kick boxer
}

\author{
T W Lloyd, M P H Tyler, A H N Roberts
}

\begin{abstract}
A 23 year old male kick boxer presented with a 24 hour history of pain and being unable to extend the interphalangeal joint of the left thumb. There was no history of trauma or any other risk factor for spontaneous rupture of the extensor pollicis longus tendon. On the previous day, he had been doing reverse press ups on the dorsum of his hands with his wrists hyperflexed as part of his training for kick boxing. At operation the extensor pollicis longus tendon was found to be divided at the level of the dorsal tubercle of the radius and was not directly repairable. The treatment was an extensor indicis proprius transfer. We suggest that the cause of the tendon rupture was direct pressure on the dorsal tubercle of the radius sustained while performing reverse press ups.

(Br J Sports Med 1998;32:178-179)
\end{abstract}

Keywords: tendon rupture; extensor pollicis longus; kick boxing

Spontaneous rupture of extensor tendons at the wrist usually affects the extensor pollicis longus, and the most common causes are fracture of the distal radius ${ }^{1}$ and rheumatoid arthritis. ${ }^{2}$ Other causes of spontaneous rupture include osteoarthitis of the radioulnar joint, ${ }^{3}$ fluoroquinolone antibiotics, especially with the concomitant use of corticosteroids, ${ }^{4}$ and certain sporting activities. Sports associated rupture of the extensor pollicis longus tendon has been reported in a 17 year old diver, ${ }^{5}$ a 69 year old tennis player, ${ }^{5}$ a 29 year old practicing judo, ${ }^{6}$ and a 19 year old woman turning the steering wheel of her car. ${ }^{7}$

Department of Plastic Surgery, Stoke Mandeville Hospital, Aylesbury, Bucks HP21 8AL, United Kingdom $\mathrm{T}$ W Lloyd M P H Tyler

A H N Roberts

Correspondence to: Mr M P H Tyler.

Accepted for publication 9 October 1997
Figure 1 The attenuated tendon divided at the level of the dorsal tubercule of the radius at the time of operation.

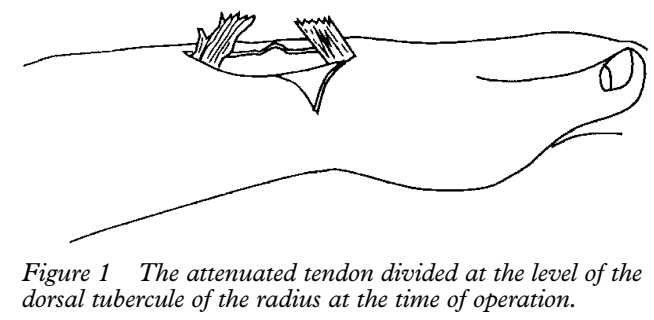

Case history

A 23 year old right hand dominant male kick boxer presented with a 24 hour history of an inability to extend the interphalangeal joint of his left thumb and with pain. On the previous day, he had been training for a national competition. Part of his training involved press ups on the dorsum of his hands with his wrists hyperflexed, and during this exercise he felt pain in his wrist. Later that evening he had pain in his left thumb which he was unable to extend fully. There was no history of antibiotic or corticosteroid use and no evidence of degenerative joint disease.

Examination showed him to be a physically fit and well built 23 year old. He was unable to extend his left thumb at the interphalangeal joint and had reduced power in extension at the metacarpophalangeal joint. A deficiency in the extensor pollicis tendon could be demonstrated at the wrist.

At surgical exploration, the extensor pollicis longus tendon was found to be divided at the level of the dorsal tubercle of the radius (fig 1). The tendon was attenuated over a considerable distance and was not suitable for direct repair. An extensor indicis proprius transfer was performed. The repair was protected with a splint for four weeks and was then mobilised. At eight weeks the patient had full range of movement of his left thumb and index finger, and at 12 weeks he returned to training.

\section{Discussion}

This unusual injury was probably a result of the reverse press ups which were part of the training routine of this patient. They involved carrying his weight on the dorsum of the hand through a flexed wrist. This is in contrast with conventional press ups where weight is carried on the palm through an extended wrist. The position of this reverse press up potentially places pressure on the dorsal tubercle of the radius. We propose that this resulted in a crush injury to the tendon causing rupture, a mechanism described by Denman. ${ }^{8}$

Attrition ruptures of tendons are rarely suitable for direct repair, and possible cases should be referred to a specialist hand unit. The extensor indicis proprius transfer is recommended by most surgeons when direct repair of the extensor pollicis longus is not possible, as it has an appropriate direction and excursion. ${ }^{9}$ 
We report this case, as rupture of the extensor pollicis longus tendon would appear to be a risk factor in sports people performing reverse press ups. We advise anyone doing these exercises to ensure that the wrists are well padded before transferring any weight to them.

1 Engkvist O, Lundborg G. Rupture of the extensor pollicis longus tendon after fracture of the lower end of the radius: a clinical and microangiographic study. Hand 1979;11:7686 .

2 Straub LR, Wilson EH Jr. Spontaneous rupture of extensor tendons in the hand associated with rheumatoid arthritis. $f$ Bone Foint Surg [Am] 1956;38:1208-17.
3 Vaughan-Jackson OJ. Rupture of extensor tendons by attrition at the inferior radio-ulnar joint. 7 Bone foint Surg [Br] tion at the inferior

4 Szarman A, Chen M, Blum MD. More on fluoroquinolone antibiotics and tendon rupture. N Engl F Med 1995;332:193. 5 Dawson WJ. Sports-induced spontaneous rupture of the extensor pollicis longus tendon. $\mathcal{F}$ Hand Surg 1992;17A: $457-8$.

6 Takami H, Takahashi S, Ando M. Traumatic rupture of the extensor tendons at the musculotendinous junction. $\mathcal{F}$ Hand Surg 1995;20A:474-7.

7 Supple KM, Zvijac JE, Janecki CJ. Spontaneous atraumatic, nonrheumatic rupture of the extensor pollicis longus tendon: a case report. F Hand Surg 1992;17A:456.

8 Denman EE. Rupture of the extensor pollicus longus: a Denman EE. Rupture of the exten
crush injury. Hand 1979;11:295-8.

9 Schneider LH, Rosenstein RG. Restoration of extensor polSchneider LH, Rosenstein RG. Restoration of extensor pol-
licis longus function by tendon transfer. Plast Reconstr Surg 1983;71:533-7.

\section{Commentary}

Specific sporting activities often demand unusual postures or high pressure to structures not normally designed for such biomechanical stress. This case identifies an unusual presentation of rupture of extensor pollicis longus, but for some time before the actual rupture, possibly a period of six to eight weeks, this patient would have had symptoms.

It would therefore be prudent for trainers, coaches, and therapists involved in this particular sport, and possibly others, to make the sportspersons themselves aware that the tendons around the hand are vulnerable, and, if they have symptoms on moving specific tendons, then they should seek advice from their coach or trainer, and, if abnormal postures are being employed, great care should be taken to avoid this particular event.

The authors have presented this well and have highlighted an unusual mode of presentation of this particular tendon rupture.

I am not entirely certain that padding the wrist would be wholly protective against rupture and would caution against relying on this technique to avoid trouble. The local anatomy is such that the tendon is almost certainly ischaemic for prolonged periods of time when the wrist is flexed. Padding would not help this. Nonetheless, I commend the authors for bringing this interesting and novel problem to our attention.

J K STANLEY 


\title{
Diagnostic pitfalls in sports related laryngeal injury
}

\author{
S D Rejali, J D C Bennett, T Upile, M P Rothera
}

\begin{abstract}
The serious nature of laryngeal trauma sustained during sporting activity can be easily overlooked. Concomitant injury should not distract from the diagnosis of potential airway problems. The effects of head and neck trauma on the airway may be delayed. The assessment and management of a patient with suspected laryngeal injury should be carried out by experienced medical practitioners.

(Br J Sports Med 1998;32:180-181)
\end{abstract}

Keywords: laryngeal trauma; supraglottic haematoma; dysphonia; airway obstruction

Every patient with a history of injury to the head, neck, or chest should be assessed for possible laryngeal injury. ${ }^{1}$ Sports related laryngeal injury is uncommon and its diagnosis difficult. $^{2}$ As laryngeal trauma may precipitate delayed airways problems, it is important that

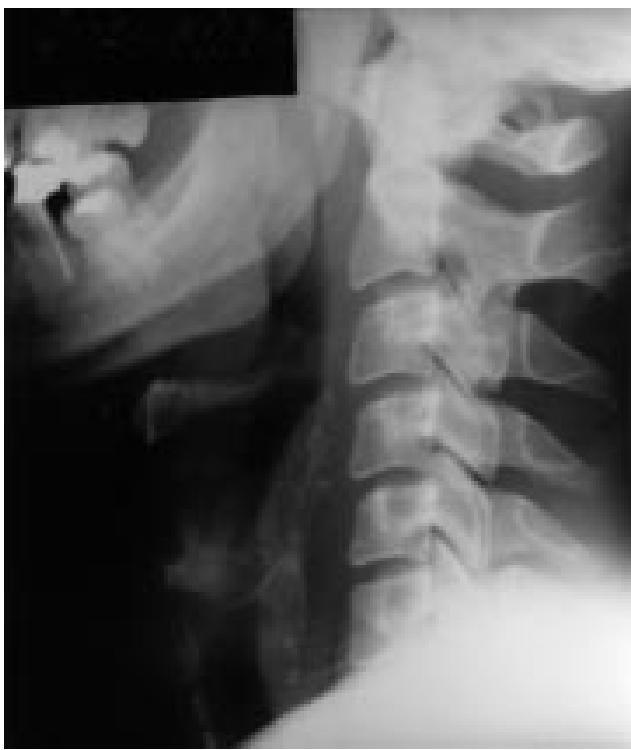

Figure 1 Normal lateral soft tissue radiograph of the neck on day of admission.

Accepted for publication 1 October 1997

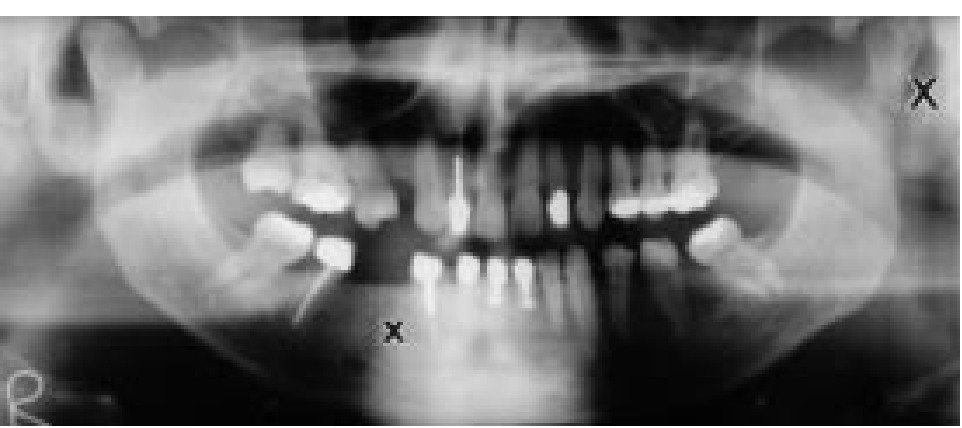

Figure 2 Radiograph of mandible with undisplaced fractures $(X)$. diagnosis is prompt and management effective. ${ }^{3}$ This case serves to illustrate some of the above and potential pitfalls in diagnosis.

\section{Case report}

A 33 year old man was seen as an emergency two hours after a game of football in which he suffered blunt injury to the mandible and neck. The injury was sustained by a direct blow to the left side of the jaw by an opponent's thigh during a mid air collision. He had neck and jaw pain exacerbated with movement and dysphagia. On examination, the left side of the neck was tender, particularly over the hyoid bone and ramus of the mandible. There was no crepitus, swelling, or bruising. Blood was present in the left external auditory meatus from a laceration in the canal but the eardrum was intact. He had no stridor. Radiographs of the soft tissues of the neck were unremarkable (fig 1 ). A radiograph of the mandible showed an undisplaced fracture at its neck on the left and a midline fracture of the body (fig 2). The patient was admitted to the hospital and spent an uneventful night. The following morning he had developed hoarseness and a short episode of dyspnoea which resolved spontaneously. Flexible nasendoscopy showed a supraglottic haematoma covering the right arytenoid cartilage causing $20 \%$ narrowing of the airway (fig 3). Repeat soft tissue $x$ ray of the neck showed a supraglottic shadow (fig 4). Treatment was started with oral prednisolone $20 \mathrm{mg}$ three times a day, analgesia, and voice rest. The patient improved over the next day and was monitored with flexible nasendoscopy for another day before being discharged with a five day course of steroids and analgesia. The fractured mandible required conservative treatment only.

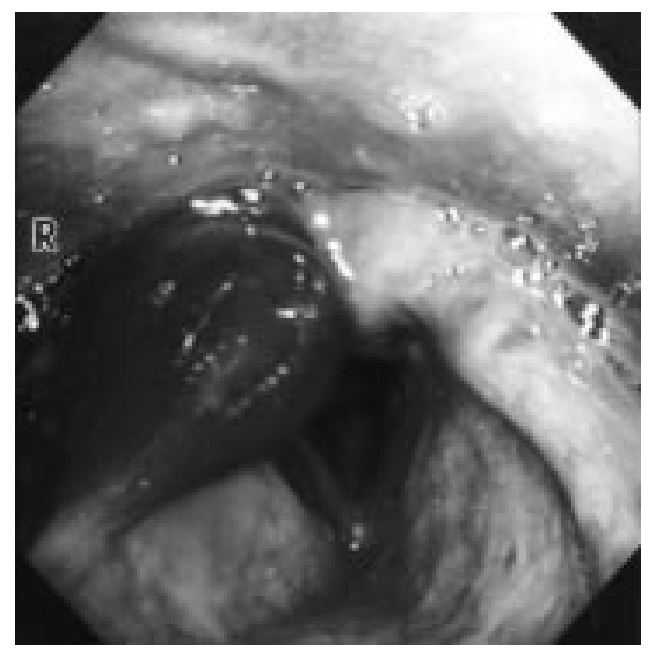

Figure 3 Endoscopic view of larynx with haematoma over the right arytenoid cartilage. 


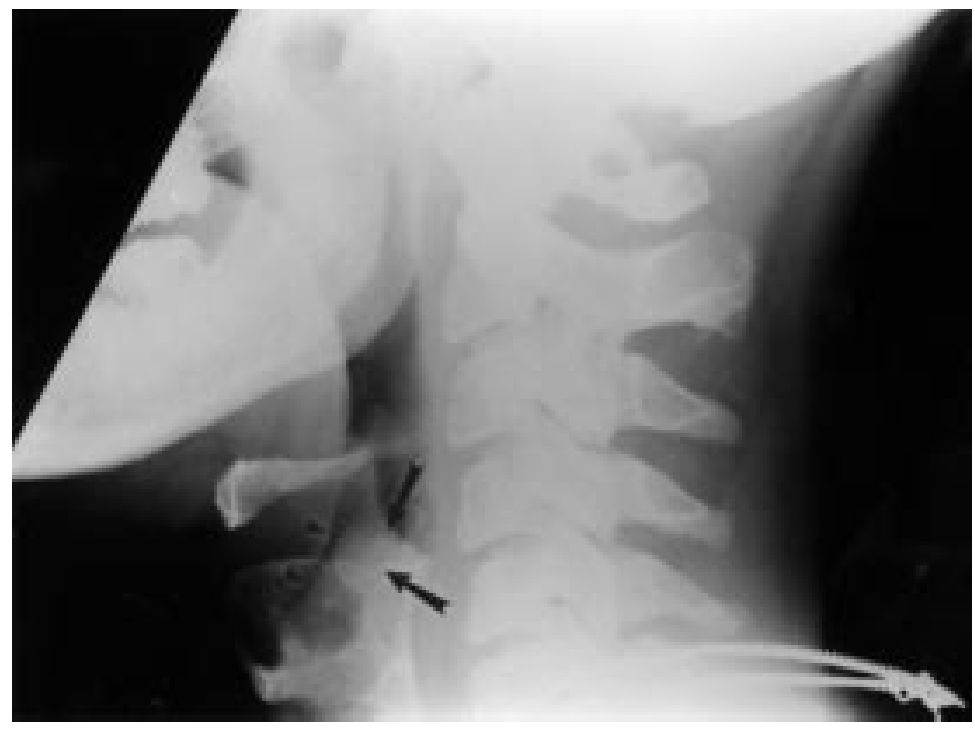

Figure 4 Soft tissue radiograph of neck. Note the outline of the supraglottic haematoma (large arrows). observation period. ${ }^{5}$ The use of steroids in laryngeal injury is controversial. Some authorities use steroids routinely claiming that, by reducing the inflammatory response and the resultant oedema, the threat to the airway is reduced, and early surgical intervention, if needed, results in improved reconstruction. ${ }^{6}$ There has been no randomised study of their benefit in conservatively treated laryngeal injury. ${ }^{7}$ Steroids used early have shown benefit in the treatment of spinal cord injury presumably by limiting oedema. ${ }^{8}$ Whether this is relevant to laryngeal injury has not been established. The policy at our unit is to use a short course of steroids with the aim of reducing mucosal oedema. It should be emphasised that close observation and timely intervention to protect the airway are the key elements in management of less serious injury. More serious laryngeal trauma may require immediate life saving manoeuvres such as intubation or establishment of a surgical airway. Later complex reconstructive laryngeal surgery may be appropriate. The association of mandibular and laryngeal injury has been reported previously. ${ }^{9}$ This can mask and/or detract from diagnosis of laryngeal injury.

In conclusion, when dealing with sports related head and neck trauma, the assessing doctor must (a) have a high index of suspicion for laryngeal trauma, (b) not be distracted from diagnosing laryngeal injury by more obvious injuries, (c) be aware that the time of maximum threat to the airway may be delayed by up to 24 hours, and (d) use a systematic method of assessment and management based on the following principles: resuscitation, accurate and thorough history and physical examination, appropriate investigations, airway monitoring, and early referral to the otolaryngologists in cases of suspected laryngeal injury.

Janet Stringfellow FRCR, Senior Registrar in Radiology, interpreted the $x$ ray findings and labelled the figures.

1 Myers EM, Iko BO. The management of acute laryngeal trauma. F Trauma 1987;27:448-52.

2 Hanft K, Palmer M, Fletcher SJ. Diagnosis and management of laryngeal trauma in sports. South Med F 1996; 89:631-3.

3 Yen PT, Lee HY, Tsai MH, et al. Clinical analysis of external laryngeal trauma. F Laryngol Otol 1994;108:221-5.

4 Ganzel TM, Munford LA. Diagnosis and management of acute laryngeal trauma. Am Surg 1989;55:303-6.

5 Schaefer SD, Close LG. Acute management of laryngeal Schaefer SD, Close LG. Acute management of laryt
trauma. Ann Otol Rhinol Laryngol 1989;98:98-104.

6 Olson N R. Surgical treatment of acute blunt laryngeal injuries. Ann Otol Rhinol Laryngol 1978;87:716-21.

7 ries. Ann Otol Rhinol Laryngol 1978;87:716-21. ries. Arch Otolaryngol Head Neck Surg 1991;117:35-9.

8 Bracken MB, Shepard MJ, Collins WF, et al. A randomised controlled trial of methyl prednisolone or naloxone in the treatment of acute spinal cord injury. $N$ Engl $f \mathrm{Med}$ 1990;322:1405-11.

9 Smith AC, Bradley PJ. Progressive dyspnea following facial injury. Br f Oral Maxillofac Surg 1986;24:28-30. appropriate management of minor haematoma is conservative with a minimum 24 hours

\section{Commentary}

One of the tenets "carved in stone" during the proper training of an otolaryngologist is that patients with closed laryngeal injuries (usually caused by karate chops or, before the days of car seat belts, steering wheel/laryngeal deceleration impact) are always admitted for overnight observation and airway monitoring. The reason cited for this is that the airway compromising swelling may be delayed for up to 24 hours. Although this article is anecdotal and not statistically at all valid, it nonetheless provides a very well illustrated example which underlines this extremely important and inviolable standard of practice. It would, of course, be difficult to find statistically viable numbers, and unethical to do a controlled trial. 


\title{
Chronic groin pain in an athlete: an unusual presentation
}

\author{
E V Williams, P Windless, S Blease, K Queen, T Jenkinson
}

\begin{abstract}
Persistent disabling groin pain in an active sportsman is a frustrating diagnostic and management problem for both the athlete and physician. After clinical examination and investigation there remains a group of patients who have unexplained groin pain, and may undergo lengthy periods of conservative management with numerous radiological investigations. Here we highlight such a case.

(Br J Sports Med 1998;32:182-183)
\end{abstract}

\section{Case report}

A 32 year old sportsman had a nine year history of intermittent right iliac fossa and right groin pain. His symptoms had initially developed after a rugby match in which there was no history of trauma. The pain would become worse in the evening after a match, especially after alcohol. It was relieved by lying flat and taking non-steroid anti-inflammatory drugs. In between the episodes he was asymptomatic, but as a result of the pain he was forced to stop playing rugby.

In the past, clinical examination had shown a tender fullness in the right iliac fossa and he was therefore investigated for possible Crohn's disease. Barium studies of the large and small bowel were, however, normal. An abdominal ultrasound scan was arranged but he failed to attend.

He was seen by a rheumatologist; examination showed no abnormality and no bony tenderness was noted. Resisted movement failed to reproduce the pain. Plain pelvic and lumbar spine $x$ rays were unremarkable, and a bone scan was reported as "normal" (fig 1). As there was no evident cause for the pain, he was referred for physiotherapy. A few months later, he presented as an emergency with an acute exacerbation. On examination, the abdomen was soft, but there was marked local tenderness in the right groin and iliac fossa, without guarding. The hernial orifices were intact. $\mathrm{He}$ was treated successfully with bed rest and analgesia. All initial investigations (blood tests, mid stream urine and abdominal $x$ ray) were normal.

An intravenous urogram showed a vague area of increasing density in the right hemipelvis (fig 2), and a normal left renal tract. Ultrasound showed gross hydronephrosis with marked cortical thining in the right pelvic kidney, and an isotope renogram
Department of

Radiology, Nevill Hall

Hospital, Abergavenny, Gwent, United

Kingdom

$S$ Blease

\section{Department of}

Urology

K Queen

Department of

Rheumatology

E V Williams

$P$ Windless

T Jenkinson

Correspondence to:

Mr E V Williams, 25

Heol-Y-Coed, Rhiwbina,

Cardiff, South Glamorgan

CF4 6HQ, United Kingdom.

Accepted for publication 25 November 1997

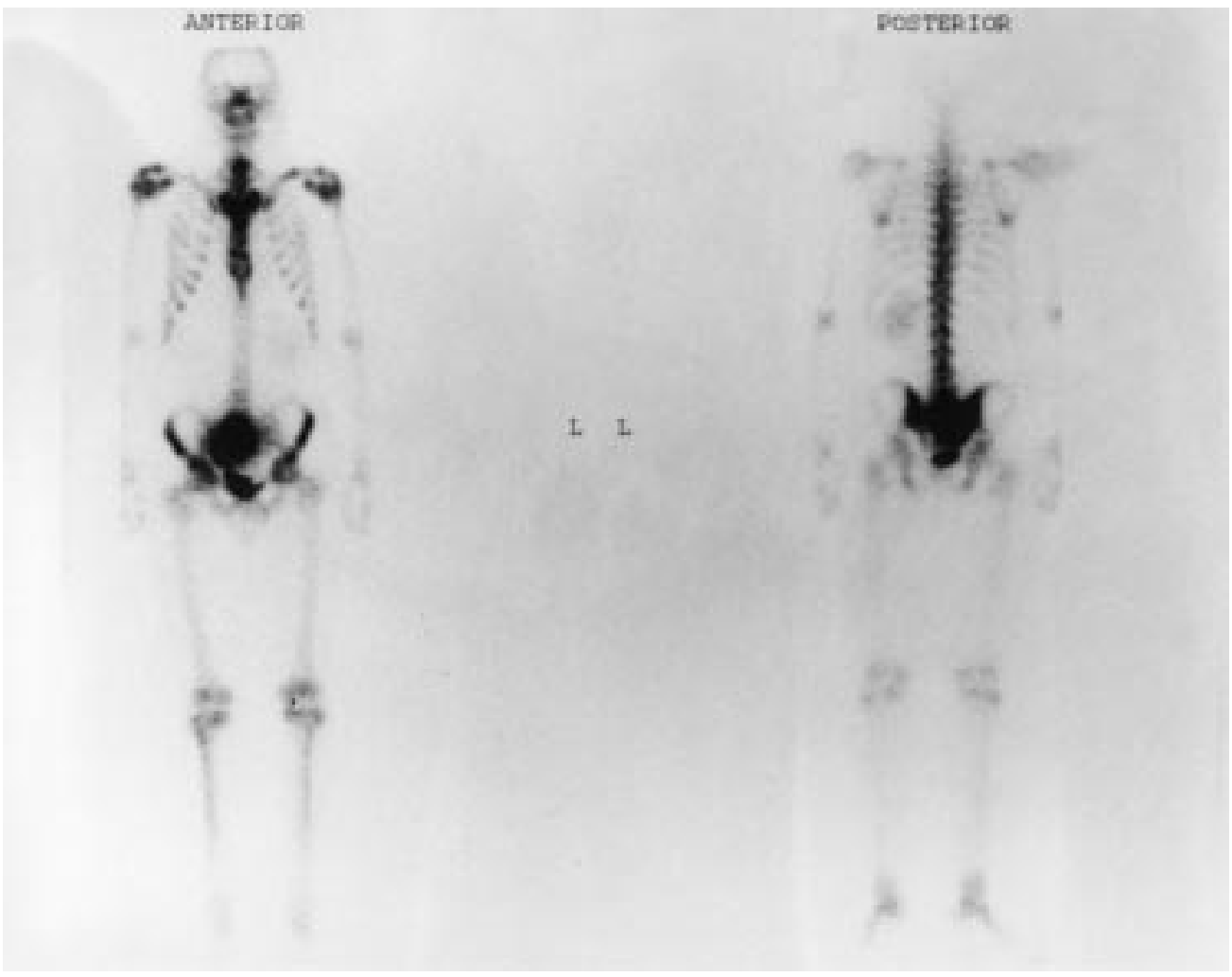

Figure 1 Isotope bone scan showing an area of increasing activity adjacent to the bladder. 


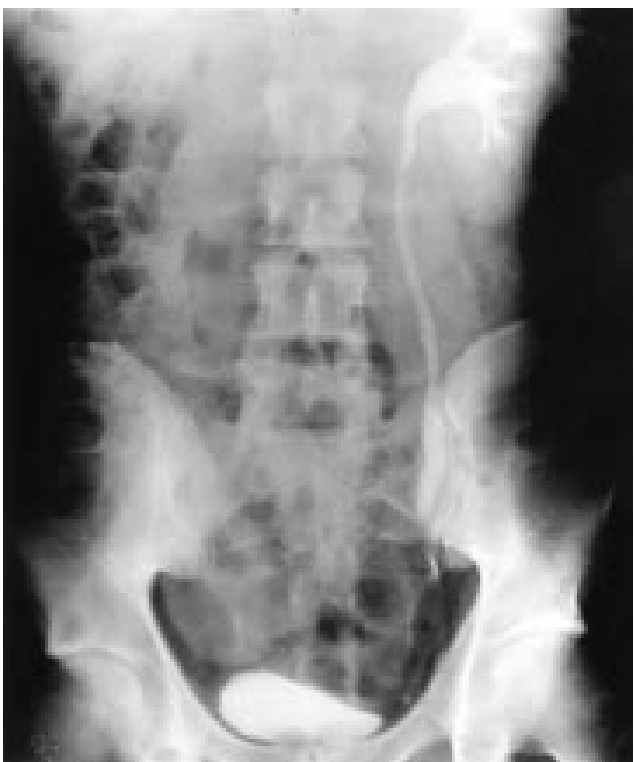

Figure 2 Intravenous urogram showing prompt excretion from the left kidney, with a nephrogram in the right hemipelvis.

diethylenetriaminepentaacetic acid (DPTA) confirmed obstruction with poor function $(5 \%)$.

He has subsequently undergone a right pelvic nephrectomy with an unremarkable recovery. Histology confirmed a hydronephrosis secondary to a pelviureteric junction (PUJ) stenosis. At six weeks follow up, he was well and asymptomatic.

\section{Discussion}

Groin pain is a common problem among sportsmen and athletes $;^{12}$ its management must be based on an accurate diagnosis. Acute conditions such as strains, hernias, and muscle ruptures are easily diagnosed and adequately treated. However, in many cases the pain develops gradually with uncharacteristic symp- toms, which may be diffuse and non-specific, making the differential diagnosis very difficult.

Defects in embryological renal development may lead to ectopic or maldeveloped renal systems. The kidneys arise from the lower pole of the urogenital ridge and migrate upwards during fetal development. Failure of migration results in a pelvic ectopic kidney. ${ }^{3}$ The problems associated with pelvic kidneys usually relate to impaired urinary drainage with secondary obstruction or stone formation. They are also more susceptible to minor trauma. In the adult, presentation is typically that of pain especially after a fluid load (Brewers kidney). An ectopic kidney must be considered if an unsuspected mass is palpated in the abdomen. Diagnosis may be made by ultrasound scan, and obstruction confirmed by intravenous urogram and isotope studies.

This case highlights three important points. Firstly, pain precipitated by a fluid load (alcohol) should make one suspect PUJ obstruction. Secondly, an ultrasound scan should be high on the list of investigations for any mass. This was arranged but the patient failed to attend. Finally, it is important to review the imaging, as errors are occasionally made in reporting. Had the bone scan been reviewed, the large area of increased activity in the right hemipelvis might not have been dismissed as bladder.

In conclusion, we present an unusual cause of abdominal and groin pain in a sportsman. It demonstrates the importance of good clinical history taking and examination in order to develop a working diagnosis and plan of investigations. Not all pains in sportsmen are necessarily related to injury.

1 Rehnstrom P, Peterson L. Groin injuries in athletes. $\mathrm{Br} \mathcal{F}$ Sports Med 1980;14:30-6.

2 Smodlaka VN. Groin pain in soccer players. Physician and Sportsmedicine 1980;8:57-61.

3 Whitfield HN, Hendry W. Congenital disorders of the kidney. In: Textbook of genito-urinary surgery, 1985;1:140-1. 